\title{
Comunicación
}

\section{DESCRIPCIÓN CLÍNICA DE LA DEMODICOSIS BOVINA (Demodex bovis) EN CÓRDOBA, COLOMBIA}

\author{
Clinical Description of Bovine Demodicosis (Demodex bovis) in Cordoba, \\ Colombia
}

\author{
José Cardona Á. ${ }^{1,4}$, Marlene Vargas V. ${ }^{3}$, Sandra Perdomo A. ${ }^{2}$
}

\section{Resumen}

Se hizo una descripción clínica y diagnóstica de la demodicosis bovina en Córdoba, Colombia, mediante un estudio descriptivo no probabilístico. Se seleccionaron 12 terneros mestizos, de ambos sexos, entre 7 a 10 meses de edad y con lesiones dermatológicas compatibles con demodicosis bovina. Las lesiones cutáneas consistieron en grave dermatitis nodular diseminada, caracterizada por su apariencia nodular y multifocal. Los nódulos eran de consistencia firme y fibrosa y con tamaños entre $3 \mathrm{~mm}$ y $1 \mathrm{~cm}$ de diámetro, ubicadas en forma bilateral a nivel de cuello y dorso. Al comprimir los nódulos, hubo salida de un material caseificado purulento, con presencia de larvas y adultos del ácaro Demodex bovis.

Palabras clave: bovino, demodicosis, Demodex bovis, dermatitis nodular

\section{AbStract}

A clinical description and diagnosis of bovine demodicosis was conducted in Cordoba, Colombia, through a descriptive and non-probabilistic study. Twelve crossbred calves of both sexes, 7 to 10 months of age with skin lesions compatible with bovine demodicosis were selected. Skin lesions consisted of severe disseminated nodular dermatitis, characterized by multifocal nodular appearance. Nodules were firm and of fibrous consistency with sizes between $3 \mathrm{~mm}$ and $1 \mathrm{~cm}$ in diameter, placed bilaterally in the neck and the back. The nodules contained caseous purulent material with presence of larvae and adult mites (Demodex bovis).

Key words: bovine, demodicosis, Demodex bovis, nodular dermatitis

\footnotetext{
${ }^{1}$ Departamento de Ciencias Pecuarias, ${ }^{2}$ Laboratorio de Patología, Universidad de Córdoba, Montería, Colombia

${ }^{3}$ Departamento de Medicina Veterinaria, Universidad Federal de Viçosa, Viçosa, Brasil

${ }^{4}$ E-mail: cardonalvarez@hotmail.com
} 


\section{INTRODUCCIÓN}

La demodicosis bovina es causada por el Demodex bovis; un ácaro blando, pequeño y en forma de cigarro. Los ácaros del género Demodex infestan los folículos pilosos y las glándulas sebáceas de todas las especies de animales domésticos, siendo especie-específicos y que en circunstancias normales, causan daños mínimos y en algunos casos, cohabitan como comensales (Wall, 2007). La importancia de la demodicosis subclínica crónica está relacionada a los daños causados en la piel y los consecuentes perjuicios en la industrialización y comercialización del cuero.

La demodicosis ha sido reportada en caninos (Izdebska, 2010; Tsai et al., 2011), felinos (Ortúñez et al., 2009), porcinos (Santarém et al., 2005), equinos (Littlewood, 1999), caprinos (Dalapati et al., 1997) y bovinos (Sakare et al., 2009; Martinelle et al., 2010); asimismo, se ha reportado en diversas especies silvestres (Vestweber et al., 1999; Gentes et al., 2007; Desch et al., 2010) y en el humano (Kulac et al., 2008).

En el bovino, la transmisión natural puede ocurrir por contacto directo entre la madre y el ternero, y por contacto entre bovinos susceptibles y altamente infectados (Silveira et al., 2012). La nutrición deficiente, el estrés fisiológico de la gestación y lactación, y la presencia de otras infecciones pueden estar asociados con brotes de demodicosis bovina (Faccini et al., 2004).

Clínicamente, la demodicosis en bovinos puede presentar lesiones cutáneas visibles y, algunas veces, estar asociada a signos clínicos como pápulas, que evolucionan a nódulos distribuidos en la superficie de los miembros, cara, cuello y la región escapular (Lemos et al., 2005); sin embargo, la enfermedad es generalmente leve y los animales pueden recuperarse en forma espontánea (Matthes, 1994; Mbuthia et al., 1994). El tamaño de los nódulos varía entre pocos milímetros hasta más de tres centímetros de diámetro, con contenido mucopurulento o caseoso y grandes cantidades de ácaros en todos los estadios del ciclo biológico (huevo, larva, protoninfa, deutoninfa y adulto) (Lemos et al., 2005). La unión de dos o más nódulos puede originar lesiones de tipo granulomatosas o exantemas costrosos, con invasión secundaria de bacterias u hongos que agravan el cuadro clínico (Faccini et al., 2004).

El diagnóstico se basa en la sintomatología clínica y la identificación del parásito al examen microscópico del material colectado de las lesiones (Silveira et al., 2012). Entre los diagnósticos diferenciales para la lesión cutánea asociada a demodicosis se deben considerar a la sarna sarcóptica (Sarcoptes scabiei var. bovis), psoróptica (Psoroptes natalensis), corióptica (Chorioptes bovis), fotosensibilización, urticaria, paraqueratosis por deficiencia de zinc y ciertas formas de dermatofilosis (Dermatophilus congolensis) (Fighera et al., 2005).

\section{Materiales y Métodos}

El estudio se llevó a cabo en el departamento de Córdoba, Colombia, a una altura de $30 \mathrm{msnm}$. La zona presenta una temperatura promedio anual de $28{ }^{\circ} \mathrm{C}$, humedad relativa de $82 \%$, precipitación media anual de 1400 $\mathrm{mm}$ y pertenece a la formación climática de bosque tropical lluvioso, con dos estaciones bien definidas (época de lluvias y época seca). El estudio de campo se realizó entre los meses de octubre y diciembre de 2010, correspondiente a la época de transición de lluvia a sequía.

El estudio fue de tipo descriptivo no probabilístico. Para este fin, se utilizaron 12 bovinos mestizos doble propósito (8 machos y 4 hembras), destetados, de 7 a 10 meses de edad, provenientes de dos explotaciones ganaderas ubicadas en áreas rurales del departamento y que presentaban lesiones nodulares, multifocales y diseminadas en la piel, compa- 


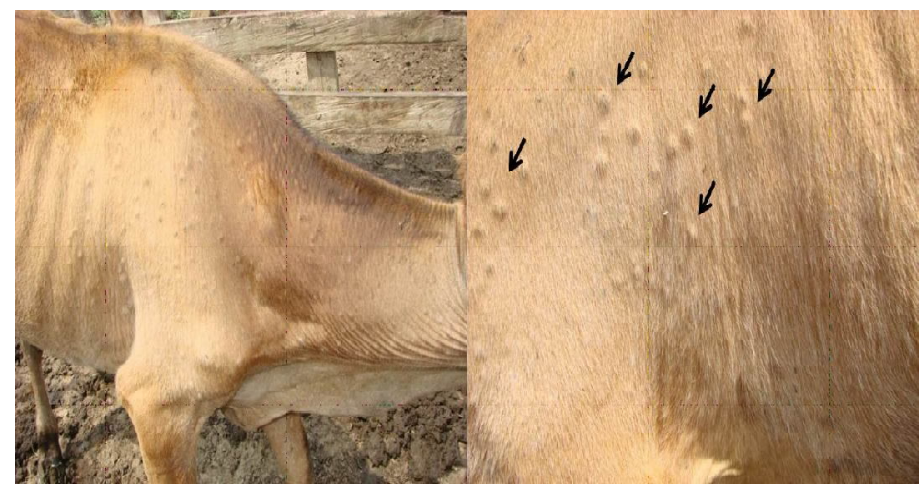

Figura 1. Dermatitis nodular multifocal y diseminada (ver las flechas) en bovinos con demodicosis

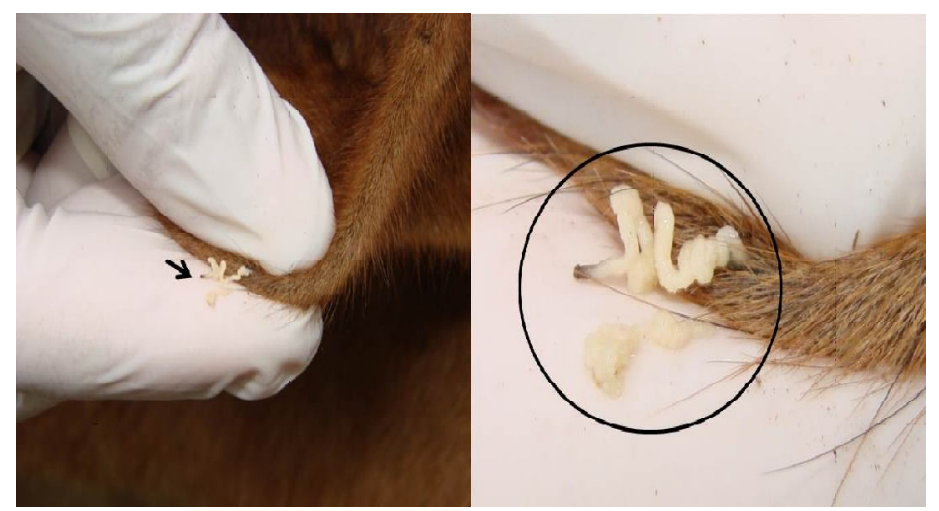

Figura 2. Compresión del nódulo parasitario (flecha), con salida de material muco-purulento y caseificado (círculo) en un bovino con demodicosis

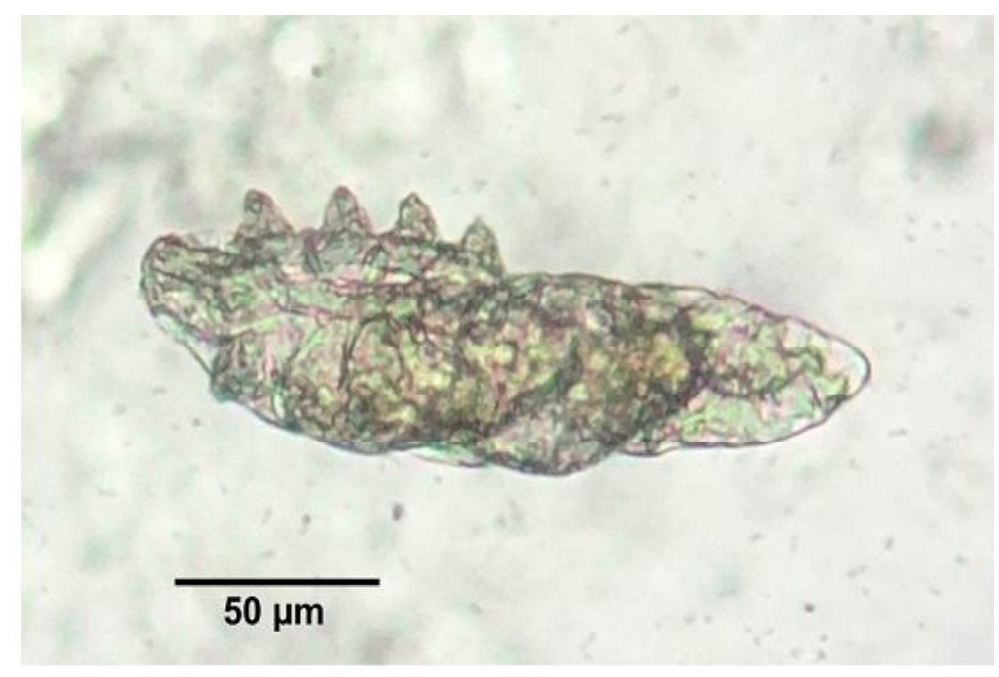

Figura 3. Demodex bovis. Nótese la característica alargada, con cuatro pares de patas ventrales a nivel del tercio anterior del cuerpo. 20x 
tibles con demodicosis bovina. Los animales no se sometieron a dolor o estrés innecesario, en cumplimiento con los principios éticos internacionales para la investigación biomédica con animales del CIOMS (Council for International Organizations of Medical Sciences) establecida por la UNESCO y la OMS (Mrad, 2006).

Se realizó una evaluación y caracterización clínica de las lesiones cutáneas, tomándose fotografías de alta definición para su posterior análisis. Asimismo, se registraron los parámetros fisiológicos de temperatura, tiempo de llenado capilar, y frecuencia ruminal, cardiaca y respiratoria. Se tomaron muestras del material mucopurulento o caseificado de las lesiones, conservadas en alcohol al $70 \%$ y llevadas al Laboratorio de Parasitología de la Facultad de Medicina Veterinaria de la Universidad de Córdoba. Allí se procedió a la observación directa con el microscopio de la presencia de ácaros del género Demodex de acuerdo con Silveira $e t$ al. (2012).

\section{Resultados y Discusión}

Las manifestaciones clínicas, las características de las lesiones en la piel y las observaciones hechas al microscopio confirmaron el diagnóstico de demodicosis en los 12 animales.

Las lesiones cutáneas consistieron en grave dermatitis nodular diseminada, caracterizada por su apariencia nodular y multifocal. Los nódulos eran de consistencia firme y fibrosa y con tamaños entre $3 \mathrm{~mm}$ y $1 \mathrm{~cm}$ de diámetro, ubicadas en forma bilateral a nivel de cuello y dorso (Fig. 1). Pérdida de condición corporal se observó en tres animales aunque los parámetros fisiológicos de todos los animales se encontraban dentro de los valores normales. A la compresión de los nódulos se observó material blanco amarillento mucopurulento y algunas veces caseificado (Fig. 2). En las todas las mues- tras de contenido de los nódulos se observaron las formas larvarias y de adultos de Demodex bovis (Fig. 3).

Las manifestaciones clínicas encontradas concuerdan con otros reportes de demodicosis (Matthes, 1994; Mbuthia et al., 1994), quienes describen lesiones cutáneas visibles desde pequeñas pápulas de $3 \mathrm{~mm}$ hasta nódulos de $3 \mathrm{~cm}$. Sin embargo, es preciso tener en cuenta la anamnesis, el tiempo de padecimiento de la enfermedad y la evolución del cuadro, pero sobretodo la observación del parásito (Silveira et al., 2012).

Los animales evaluados en el presente estudio correspondieron a terneros doble propósito destetados, de 7 a 10 meses de edad, de ambos sexos, especialmente machos, por lo que podría inferirse que el cambio de época (lluvias a sequía), así como el proceso de destete en algunos de ellos, influyeron en la presentación de estrés e inmunosupresión, favoreciendo su presentación; sin embargo, Faccini et al. (2004) reportaron mayor frecuencia de presentación en animales de 36 a 47 meses de edad, especialmente en hembras. No se encontraron datos en la literatura consultada sobre la frecuencia de presentación de la demodicosis en el departamento de Córdoba y Colombia.

\section{Literatura Citada}

1. Dalapati M, Bhowmik M, Sarkar S, Sasma N. 1997. Demodicosis of goats: haematological and biochemical changes. Trop Anim Hlth Prod 4: 240-242.

2. Desch C, Andrews J, Baeten L, Hol$\operatorname{der} Z$, Powers J, Weber D, Ballweber L. 2010. New records of hair follicle mites (Demodecidae) from North American Cervidae. J Wildlife Dis 46: 585-590.

3. Faccini J, Santos A, Bechara G 2004. Demodicose bovina no Estado da Paraíba. Pesq Vet Bras 24: 149-153. 
4. Fighera R, Souza T, Lombardo de Barros $C$. 2005. Lesões de pele em bovinos com doença granulomatosa sistêmica associada ao pastoreio de ervilhaca ( $\mathrm{Vi}$ cia spp). Ciência Rural 35: 406-411.

5. Gentes M, Proctor H, Wobeser G. 2007. Demodicosis in a mule deer (Odocoileus hemionus hemionus) from Saskatchewan, Canada. J Wildlife Dis 43: 758-761.

6. Izdebska J. 2010. Demodex sp. (acari, demodecidae) and demodecosis in dogs: characteristics, symptoms, occurrence. Bull Vet Inst Pulawy 54: 335-338.

7. Kulac M, Hakký I, Karaca S, Cetinkaya Z. 2008. Clinical importance of Demodex folliculorum in patients receiving phototherapy. Int J Dermatol 47: 72-77.

8. Lemos L, Santos A, Sales L, Silveira L, Carvalho E. 2005. Demodicose bovina: relato de caso. Arq Bras Med Vet Zootec 57: 732-735.

9. Littlewood L. 1999. Control of ectoparasites in horses. In Practice 21: 418-424.

10. Martinelle L, Dal Pozzo F, Losson B, Sarradin P, Saegerman C. 2010. Cas de démodécie chez deux veaux de race holstein. Ann Méd Vét 154: 61-64.

11. Matthes H. 1994. Investigations of pathogenesis of cattle demodicosis: sites of predilection, habitat and dynamics of demodectic nodules. Vet Parasitol 53: 283-291.

12. Mbuthia PG, Kariuk DI, Mulei CM. 1994. Generalized demodicosis in a Friesian heifer from a zero-grazing unit. Vet Parasitol 51: 337-343.
13. Mrad A. 2006. Ética en la investigación con modelos animales experimentales. Alternativas y las 3 RS de Russel. Una responsabilidad y un compromiso ético que nos compete a todos. Rev Colomb Bioética 1: 163-184.

14. Ortúñez A, Verde M, Navarro L, Real L, Vilela C. 2009. Demodicosis felina: a propósito de tres casos clínicos. Clin Vet Peq Anim 29: 165-171.

15. Sakare M, Bhicane A, Lengare A, Awaz K. 2009. Demodectic mange in calves. A clinical report. Intas Polivet 10: 273-274.

16. Santarém V, Farias M, Tostes R. 2005. Demodectic mange in fattening pigs in Sao Paulo, Brazil. Vet Parasitol 131: 169171.

17. Silveira R, Ribeiro R, CruzA, Almeida L, Carvalho E. 2012. Demodicose suína no norte do estado do Rio de Janeiro: relato de caso. Arq Bras Med Vet Zootec 64: 555-558.

18. Tsai Y, Chung W, Wang L, Ju Y, Hong C, Tsai Y, Li Y, Wu Y. 2011. The dog mite, Demodex canis: Prevalence, fungal co-infection, reactions to light, and hair follicle apoptosis. J Insect Sci 2(76): 1-13.

19. Vestweber J, Ridley R, Nietfeld J, Wilkerson M. 1999. Demodicosis in an American bison. Can Vet J 40: 417-418.

20. Wall R. 2007. Ectoparasites: Future challenges in a changing world. Vet Parasitol 148: 62-74.

21. Wolhuter J, Bengis R, Reilly B, Cross $P$. 2009. Clinical demodicosis in African buffalo (Syncerus caffer) in the Kruger National Park. J Wildlife Dis 45: 502-504. 